\title{
ON MINIMAL SURFACES SATISFYING THE OMORI-YAU PRINCIPLE
}

\author{
ALBERT BORBÉLY
}

(Received 8 August 2010)

\begin{abstract}
Complete minimal immersions satisfying the Omori-Yau maximum principle are investigated. It is shown that the limit set of a proper immersion into a convex set must be the whole boundary of the convex set. In case of a nonproper and nonplanar immersion we prove that the convex hull of the immersion is a half-space or $\mathbb{R}^{3}$.
\end{abstract}

2010 Mathematics subject classification: primary 53C42.

Keywords and phrases: minimal surface, Omori-Yau maximum principle.

\section{Introduction}

Let $M$ be a complete Riemannian manifold. We say that $M$ satisfies the Omori-Yau principle if the following holds.

OMORI-YAU PRINCIPLE. Let $f: M \rightarrow \mathbb{R}$ be a continuous function which is bounded from below (or above, respectively). Let us also assume that $f$ is $C^{2}$ on the set $\{f<\inf f+\epsilon\}$ (or on $\{f>\sup f-\epsilon\}$, respectively) for some $\epsilon>0$. Then there is a sequence $Q_{n} \in M$ such that:

(1) $\quad f\left(Q_{n}\right) \rightarrow \inf f$ (or $f\left(Q_{n}\right) \rightarrow \sup f$, respectively);

(2) $\left|\nabla f\left(Q_{n}\right)\right| \rightarrow 0$;

(3) $\lim \inf \Delta f\left(Q_{n}\right) \geq 0$ (or lim $\sup \Delta f\left(Q_{n}\right) \leq 0$, respectively).

REMARK 1.1. It is customary to require $f$ to be $C^{2}$ everywhere but it is easy to see that it matters only when $f$ is close to its infimum (or supremum, respectively). Since it is obviously true for compact manifolds, this refers to the behavior at infinity of noncompact manifolds.

This principle turned out to be a powerful tool in geometric analysis (see, for instance, $[3,5,11,14]$ for a recent application).

There are various curvature conditions that force the manifold to satisfy the OmoriYau principle. It was shown by Omori [10] that if $M$ is a complete manifold with

(C) 2011 Australian Mathematical Publishing Association Inc. 0004-9727/2011 \$16.00 
sectional curvatures bounded from below, then $M$ satisfies the Omori-Yau principle. This was generalized by Yau [13] showing that if $M$ is a complete manifold with Ricci curvature bounded from below, then $M$ satisfies the Omori-Yau principle. Recently a further generalization was given. It was shown in [11] and also in [1] that if the Ricci curvature does not converge to $-\infty$ too fast, then the manifold satisfies the Omori-Yau principle. Roughly speaking, one can consider the Omori-Yau principle instead of a growth condition on the Ricci curvature.

After the discovery of a complete minimal immersion $f: \mathbb{D} \rightarrow \mathbb{B}[9]$, where $\mathbb{D} \subset \mathbb{R}^{2}$ is the unit disk and $\mathbb{B} \subset \mathbb{R}^{3}$ is the unit ball, the attention turned towards proper minimal immersions $f: \mathbb{D} \rightarrow D$, where $D$ is some convex body. In a series of papers [6-8] the existence of such immersions was shown and their limit sets were investigated. It was proved in [8] that the limit set can be a small part of $\partial D$.

For a proper minimal immersion $\varphi: m \rightarrow D$, where $D$ is an open convex body, we define the limit set of $\varphi$ as $\varphi(\partial M)=\operatorname{Closure}(\varphi(M))-\varphi(M)$. It is obvious that $\varphi$ is proper if, and only if, $\varphi(\partial M) \subset \partial D$.

TheOREM 1.2 (Martin and Morales). Let D be a regular strictly convex bounded domain of $\mathbb{R}^{3}$, and consider a regular Jordan curve $\Gamma$. Then, for each $\epsilon>0$, there is a complete proper minimal immersion $f_{\Gamma, \epsilon}: \mathbb{D} \rightarrow D$ satisfying that the Hausdorff distance $\delta^{H}\left(f_{\Gamma, \epsilon}(\partial \mathbb{D}), \Gamma\right)<\epsilon$, where $f_{\Gamma, \epsilon}(\partial \mathbb{D})$ represents the limit set of the minimal $\operatorname{disk} f_{\Gamma, \epsilon}(\mathbb{D})$.

Our first result says that this cannot happen if the minimal surface satisfies the Omori-Yau principle. In this case, the limit set must be the whole boundary $\partial D$.

TheOREM 1.3. Let $D \subset \mathbb{R}^{3}$ be a convex open set with boundary $\partial D$. Let $f: M \rightarrow D$ be a complete proper nonplanar minimal immersion. If $M$ satisfies the Omori-Yau principle, then the limit set of $f$ is $\partial D$.

REMARK 1.4. The convex set $D$ in the theorem is not necessarily bounded. It can be a half-space for example.

Our second result concerns the convex hull of a complete minimal immersion. This area is centred around the Calabi-Yau conjectures which concern the convex hull of minimal surfaces. A recent overview can be found in [2].

A theorem of Xavier [12] states that if a complete nonplanar minimal surface has bounded sectional curvature, then the convex hull of the surface is $\mathbb{R}^{3}$. Replacing the curvature bound with a topological condition, Hoffman and Meeks [4] proved later that the convex hull of a properly immersed nonplanar complete minimal surface is $\mathbb{R}^{3}$. These results are known as half-space theorems in the literature since these surfaces cannot be contained in a half-space. It was shown recently by Colding and Minicozzi [2] that the convex hull of a nonplanar complete embedded minimal disk is $\mathbb{R}^{3}$.

It is natural to ask whether there are other conditions that would imply a half-space theorem. In connection with this, we prove a somewhat weaker version. 
THEOREM 1.5. Let $f: N \rightarrow \mathbb{R}^{3}$ be a complete minimal immersion satisfying the Omori-Yau principle. Then $f(N)$ cannot be in a wedge.

It would be interesting to know if the half-space theorem is true for complete minimal immersions satisfying the Omori-Yau principle.

\section{Proof of Theorem 1.5}

PROOF. Let us assume that the statement is not true, and there is a complete minimal immersion $f: N \rightarrow \mathbb{R}^{3}$ satisfying the Omori-Yau principle and a wedge $W$ containing $f(N)$. Without loss of generality we can assume that the edge of the wedge is the $z$-axis and that the wedge is symmetric with respect to the $y z$-plane. That is, $W=\left\{(x, y, z) \in \mathbb{R}^{3}: y<-a|x|\right\}$ for some $a>0$. Let $V=\left\{(x, y) \in \mathbb{R}^{2}: y<-a|x|\right\}$ be the intersection of $W$ with the $x y$-plane. By translating the surface in the $z$-direction if necessary we may further assume that the $x y$-plane contains a point $P=\left(x_{p}, y_{p}, 0\right)$ of the surface $f(N)$.

Choose a point $O=\left(0,-y_{0},\right)$ on the negative $y$-axis such that $-y_{0}<y_{p}$ and the circle centred around $O$ and passing through $P$ intersects the boundary of $V$. By shortening the radius slightly we obtain a disk $S$ in the $x y$-plane centred at $O$ with radius $R$ such that $V-S$ has two connected components $V_{1}$ and $V_{2}$. We denote the bounded component by $V_{1}$ and the unbounded by $V_{2}$. From the above it is clear that the point $P \in V_{1}$.

Let $W_{1}=V_{1} \times\{z$-axis $\}, W_{2}=V_{2} \times\{z$-axis $\}$, and let $r: \mathbb{R}^{3} \rightarrow \mathbb{R}$ be the distance function from the line $L$ passing through $O$ and parallel with the $z$-axis.

The Hessian of $r$ has two eigenvectors (one is in the radial direction the other is parallel to the $z$-axis) with zero eigenvalues and one eigenvector (tangent to the cylinder around $L$ and orthogonal to the $z$-axis) with a positive eigenvalue which is the reciprocal of the distance from the line $L$. Therefore we have

$$
\Delta r(Q)=1 / \operatorname{dist}(Q, L) .
$$

We define $g: N \rightarrow \mathbb{R}$ as follows:

$$
g(Q)= \begin{cases}r(f(Q)) & \text { if } f(Q) \notin W_{2}, \\ R & \text { if } f(Q) \in W_{2} .\end{cases}
$$

This means that $g$ is basically the restriction of $r$ to $f(N)$, except for that part of the surface that lies in $W_{2}$. There $g$ is constant. The distance function from the line $L$ is bounded by $y_{0}$ on $W_{1}$; therefore $g$ is bounded from above. Although $g$ is not smooth along the intersection of the surface with the boundary of $W_{2}$, we can modify it slightly along the intersection to make it smooth everywhere. Since the rest of the argument involves only the part of the surface that lies in $W_{1}$ this will cause no problem.

We will calculate $\nabla g$ and $\Delta g$ as in [1]. 
For simplicity let us identify $N$ with $g(N)$. For a point $Q \in N$ let $\alpha(Q)$ be the angle between the tangent plane of the surface $T_{Q} N$ and the tangent plane to the level surface of the distance function $r$ at the point $Q$.

We have $\nabla g=\left.\nabla r\right|_{N}=(\nabla r)^{T}$, where $(\nabla r)^{T}$ means the tangential part of $\nabla r$. Therefore

$$
|\nabla g(Q)|=\sin (\alpha(Q)) .
$$

Next we deal with the Laplacian. Let $Q \in N$ such that $Q \notin W_{2}$. Then for the tangent vectors $X, Y \in T_{Q} N$ we have

$$
\begin{aligned}
\operatorname{Hess}\left(r_{\mid N}\right)(X, Y)=\left\langle\tilde{\nabla}_{X} \nabla\left(r_{\mid N}\right), Y\right\rangle & =\left\langle\nabla_{X}\left(\nabla r-(\nabla r)^{N}\right), Y\right\rangle \\
& =\operatorname{Hess} r(X, Y)-\left\langle\nabla_{X}(\nabla r)^{N}, Y\right\rangle .
\end{aligned}
$$

Since the normal component $(\nabla r)^{N}$ of the gradient is orthogonal to $N$ we have

$$
\left\langle\nabla_{X}(\nabla r)^{N}, Y\right\rangle=-\left\langle(\nabla r)^{N}, \nabla_{X} Y\right\rangle=\left\langle(\nabla r)^{N}, A(X, Y)\right\rangle,
$$

where $A(X, Y)=-\left(\nabla_{X} Y\right)^{N}$ denotes the vector-valued second fundamental form of $N$ and $\nabla$ is the connection on $\mathbb{R}^{3}$.

Combining this with the equality above, we have

$$
\text { Hess } g(X, Y)=\operatorname{Hess} r(X, Y)-\left\langle(\nabla r)^{N}, A(X, Y)\right\rangle \text {. }
$$

Since $N$ is minimal Trace $A=0$. Taking trace in $T_{Q} N$ we have

$$
\Delta g=\text { Trace }(\text { Hess } r) \text {, }
$$

where Trace(Hess $r$ ) is not necessarily the same as $\Delta r$, since we took trace in $T_{Q} N$ only. Projecting the tangent plane $T_{Q} N$ to the plane orthogonal to $\nabla r$ we can conclude that

$$
\Delta g=\operatorname{Trace}(\operatorname{Hess} r) \geq \cos (\alpha(Q)) \Delta r(Q) .
$$

To reach a contradiction let $Q_{n} \in N$ be a sequence such that $g\left(Q_{n}\right) \rightarrow \sup g$ and $\left|\nabla g\left(Q_{n}\right)\right| \rightarrow 0$. From the construction of the function $g$ it follows that $Q_{n} \in W_{1}$ for sufficiently large $n$. Since $r$ is bounded by $y_{0}$ on $W_{1}$, formula (2.1) implies that

$$
\Delta r\left(Q_{n}\right)>\frac{1}{y_{0}} \text {. }
$$

From $\left|\nabla g\left(Q_{n}\right)\right| \rightarrow 0$ we conclude that $\sin \left(\alpha\left(Q_{n}\right)\right) \rightarrow 0$, and so $\cos \left(\alpha\left(Q_{n}\right)\right) \rightarrow 1$. Combining this with (2.5), we obtain that

$$
\lim \sup \Delta f\left(Q_{n}\right) \geq 1 / y_{0} .
$$

This contradicts the Omori-Yau principle and the proof is complete.

\section{Proof of Theorem 1.3}

The proof combines the catenoid trick of Hoffman and Meeks [4] with the observation that the distance function to a minimal surface has negative Laplacian near the surface. 
The following fact will be used later. Let $N$ be an embedded minimal surface and $p \in N$ be an arbitrary point. Let $q$ be a point on the normal line through $p$ at distance $d$ from $N$. If $\pm \lambda$ are the principal curvatures of $N$ at $p$ and $d<1 / \lambda$, then the principal curvatures of the parallel surface to $N$ through $q$ are given as

$$
-\frac{\lambda}{1-\lambda d} \text { and } \frac{\lambda}{1-\lambda d}
$$

PROOF OF THEOREM 1.3. We argue by contradiction. Let us assume that the statement of the theorem is not true. Let $P \in \partial D$ be a point that is not a limit point of $f$. We will show that in this case the surface cannot satisfy the Omori-Yau principle.

Let $S_{P}$ be a supporting hyperplane of $D$ through $P$. It is a straightforward consequence of the maximum principle that $S_{P}$ is disjoint from $f(M)$. Translate $S_{P}$ towards the surface until it 'touches' the surface and denote this hyperplane (which is not necessarily different from $S_{P}$ ) by $S$. Again, it is clear that $S$ is disjoint from $f(M)$ but any translate of $S$ towards the surface will intersect $f(M)$.

We have two possibilities. If $S \neq S_{P}$, then $S \cap D \neq \emptyset$ and let $P^{\prime}$ be an arbitrary point in $S \cap D$. Since $P^{\prime} \in S \cap D$ is in $D$, the properness of the immersion implies that it cannot be a limit point of $f$, and since it is on $S$ the surface $f(M)$ cannot pass through it, unless $f(M)$ is a plane, which contradicts the assumptions of the theorem. Therefore, there is a small $\epsilon>0$ such that $B\left(P^{\prime}, \epsilon\right) \cap f(M)=\emptyset$, where $B\left(P^{\prime}, \epsilon\right)$ denotes the open ball around $P^{\prime}$ with radius $\epsilon$.

We have the same situation if $S=S_{P}$. From the assumption that $P$ is not a limit point of $f$ we conclude that there is small $\epsilon>0$ such that $B(P, \epsilon) \cap f(M)=\emptyset$.

In either case, we have a hyperplane $S$ and a point $P \in S$ satisfying the following:

$$
\begin{gathered}
S \cap f(M)=\emptyset ; \\
\operatorname{dist}(S, f(M))=0 ; \\
\text { there is an } \epsilon>0 \text { such that } B(P, 4 \epsilon) \cap f(M)=\emptyset .
\end{gathered}
$$

Without loss of generality, we may assume that $f(M)$ is in the upper half-space $H=\{(x, y, z): z>0\}, S=\{z=0\}$ and $P$ is the origin. Similarly to [4], let $C$ be the solid half-catenoid $\left\{(x, y, z): x^{2}+y^{2} \leq \cosh ^{2}(z), z \leq 0\right\}$ and let $C_{t}$ be the homothetic shrinking of $C$ by $t, 0<t \leq 1$. We remark that $\partial C_{t}$ converges to $S-\{P\}$ as $t$ tends to 0 .

From the assumptions above it is clear that $\operatorname{dist}\left(C_{\epsilon}, f(M)\right)>0$. Therefore there is a small $0<\eta<\epsilon$ such that $\operatorname{dist}\left(f_{\eta}(M), C_{\epsilon}\right)>0$, where $f_{\eta}(Q)=f(Q)-(0,0, \eta)$, that is, $f_{\eta}(M)$ is the downward translation of $f(M)$ by $\eta$.

We let $t$ vary from $\epsilon$ to 0 until $C_{t}$ 'hits' the surface $f_{\eta}(M)$. To be more precise, consider the set $T=\left\{t \in(0, \epsilon]: \operatorname{dist}\left(f_{\eta}(M), C_{t}\right)=0\right\}$.

Since $f_{\eta}(M)$ has points below the plane $S$ and $C_{t}$ converges to $S$ as $t \rightarrow 0$, then $T$ is not empty. Set $t_{0}=\sup T$. Clearly $0<t_{0}<\epsilon$ and $\operatorname{dist}\left(f_{\eta}(M), C_{t_{0}}\right)=0$.

The part of $C_{t_{0}}$ that is above the plane $S_{2 \eta}$ (where $S_{2 \eta}$ is the downward translation of $S$ by $2 \eta$ ) is compact. By slightly increasing $t_{0}$ we can find a $t^{*} \in\left(t_{0}, \epsilon\right]$ 
such that:

$$
\begin{aligned}
& \qquad<\operatorname{dist}\left(f_{\eta}(M), C_{t^{*}}\right)=\delta<\epsilon ; \\
& \text { all principal curvatures of } \partial C_{t^{*}} \text { are less than } \frac{1}{2 \delta} .
\end{aligned}
$$

As in the previous section, let $r=\operatorname{dist}\left(\cdot, C_{t^{*}}\right)$ be the distance function from $C_{t^{*}}$, $g: M \rightarrow \mathbb{R}$ be the restriction of $r$ to $f_{\eta}(M)\left(g(Q)=\operatorname{dist}\left(C_{t^{*}}, f_{\eta}(Q)\right)\right.$ and $\alpha(Q)$ be the angle between the tangent planes of $f_{\eta}(M)$ and of the level surface of the distance function at $f_{\eta}(Q)$.

The function $g$ is not smooth on the whole of $M$ since $\partial C_{t^{*}}$ is not a smooth surface but it will be smooth on the set $\{g<2 \delta\}$. Let $Q \in\{g<2 \delta\}$ and let $R \in \partial C_{t^{*}}$ be a closest point to $f(Q)-\eta \in f(M)_{\eta}$. From the relations of $\epsilon, \eta$ and $\delta$ one concludes that $R$ cannot be on the plane $S$, therefore it is on the half-catenoid. As a result it is unique and the distance function and therefore $g$ are smooth near $Q$.

The gradients of $g$ and $r$ are related as follows:

$$
|\nabla g(Q)|=\sin (\alpha(Q))|\nabla r|=\sin (\alpha(Q)),
$$

since the gradient of the distance function is a unit vector.

As in the previous section, the hessian of $g$ and $r$ are related by formula (2.5) and the nonzero eigenvalues of the hessian of the distance function $r$ are given in (3.1). Combining the two and taking into account the angle between the tangent spaces, we obtain

$$
\begin{aligned}
\Delta g(Q) & \leq \frac{\lambda}{1+\lambda g(Q)}-\frac{\cos (\alpha(Q)) \lambda}{1-\lambda g(Q)} \\
& =\frac{(1-\cos (\alpha(Q))) \lambda-(1+\cos (\alpha(Q))) \lambda^{2} g(Q)}{1-(\lambda g(Q))^{2}}
\end{aligned}
$$

where $\delta<g(Q)=\operatorname{dist}\left(f_{\eta}(Q), C_{t^{*}}\right)<2 \delta$ and $\lambda$ denotes the positive principal curvature of the surface $\partial C_{t^{*}}$ at the point $R$.

Let $Q_{n} \in M$ be a sequence such that $g\left(Q_{n}\right) \rightarrow \delta$ and $\left|\nabla g\left(Q_{n}\right)\right| \rightarrow 0$. Denote by $R_{n} \in C_{t^{*}}$ the closest point to $f_{\eta}\left(Q_{n}\right)$ on the surface $\partial C_{t^{*}}$ and by $\lambda_{n}>0$ the positive principal curvature of $\partial C_{t^{*}}$ at $R_{n}$. From (3.2) we conclude that $\sin \left(\alpha\left(Q_{n}\right)\right) \rightarrow 0$ therefore $\cos \left(\alpha\left(Q_{n}\right)\right) \rightarrow 1$. Putting this information into (3.5), we obtain that

$$
\lim \sup \Delta g\left(Q_{n}\right) \leq \frac{-2 \lambda^{2} \delta}{1-(\lambda \delta)^{2}}<0,
$$

where $\lambda=\lim \lambda_{n}$. In view of (3.3b) we also have $\delta<1 / \lambda$.

This implies that

$$
\lim \inf \Delta g\left(Q_{n}\right)<0,
$$

which contradicts the Omori-Yau principle and concludes the proof of Theorem 1.3. $\square$

\section{Acknowledgement}

I would like to thank the referee for the careful reading of the manuscript and for the helpful suggestions. 


\section{References}

[1] A. Borbely, 'Immersion of manifolds with unbounded image and a modified maximum principle of Yau', Bull. Aust. Math. Soc. 78 (2008), 285-291.

[2] T. H. Colding and W. P. Minicozzi II, 'The Calabi-Yau conjectures for embedded surfaces', Ann. of Math. (2) 167 (2008), 211-243.

[3] F. Fontenele and F. Xavier, 'Good shadows, dynamics, and convex hulls', Asian J. Math. 15 (2011), 9-32.

[4] D. Hoffman and W. H. Meeks III, 'The strong halfspace theorem for minimal surfaces', Invent. Math. 101 (1990), 373-377.

[5] L. Jorge and D. Koutroufiotis, 'An estimate for the curvature of bounded submanifolds', Amer. J. Math. 103 (1981), 711-725.

[6] F. Martin, W. H. Meeks III and N. Nadirashvili, 'Bounded domains which are universal for minimal surfaces', Amer. J. Math. 129 (2007), 455-461.

[7] F. Martin and S. Morales, 'On the asymptotic behavior of a complete bounded minimal surface in $\mathbb{R}^{3}$, Trans. Amer. Math. Soc. 356 (2004), 3985-3994.

[8] F. Martin and S. Morales, 'Complete proper minimal surfaces in convex bodies of $\mathbb{R}^{3}$ (II): the behavior of the limit set', Comment. Math. Helv. 81 (2006), 699-725.

[9] N. Nadirashvili, 'Hadamard's and Calabi-Yau's conjectures on negatively curved minimal surfaces', Invent. Math. 126 (1996), 457-465.

[10] H. Omori, 'Isometric immersions of Riemannian manifolds', J. Math. Soc. Japan 19 (1967), 205-214.

[11] A. Ratto, M. Rigoli and A. G. Setti, 'On the Omori-Yau maximum principle and its applications to differential equations and geometry', J. Funct. Anal. 134 (1995), 486-510.

[12] F. Xavier, 'Convex hulls of complete minimal surfaces', Math. Ann. 269 (1984), 179-182.

[13] S. T. Yau, 'Harmonic functions on complete Riemannian manifolds', Comm. Pure Appl. Math. 28 (1975), 201-228.

[14] S. T. Yau, 'A generalized Schwarz lemma for Kahler manifolds', Amer. J. Math. 100 (1978), 197-203.

\section{ALBERT BORBÉLY, Department of Mathematics, Faculty of Science, Kuwait University, PO Box 5969, Safat 13060, Kuwait e-mail: borbely@ sci.kuniv.edu.kw}

